\title{
Philosophiques
}

Jean-François Filion, Dialectique et matière. La conceptualité inconsciente des processus inorganiques dans la Philosophie de la nature (1830) de Hegel, Québec, Les Presses de l'Université Laval, 2007, 346 p.

\section{Danic Parenteau}

Volume 34, numéro 2, automne 2007

URI : https://id.erudit.org/iderudit/017431ar

DOI : https://doi.org/10.7202/017431ar

Aller au sommaire du numéro

Éditeur(s)

Société de philosophie du Québec

ISSN

0316-2923 (imprimé)

1492-1391 (numérique)

Découvrir la revue

Citer ce compte rendu

Parenteau, D. (2007). Compte rendu de [Jean-François Filion, Dialectique et matière. La conceptualité inconsciente des processus inorganiques dans la Philosophie de la nature (1830) de Hegel, Québec, Les Presses de l'Université Laval, 2007, 346 p.] Philosophiques, 34(2), 425-427.

https://doi.org/10.7202/017431ar d'utilisation que vous pouvez consulter en ligne. 
souvent floues : concepts non esthétiques, esthétiques et évaluatifs (p. 59). La théorie de Sibley repose sur une analyse du rapport qu'ils entretiennent les uns aux autres et comporte le mérite de mieux répondre aux critiques de relativisme qu'on pouvait faire aux théories évoquées précédemment (p. 68). Mais elle n'est pas exempte non plus de difficultés, soulignons par exemple le caractère culturellement relatif des concepts esthétiques (p. 71).

La seconde partie de l'ouvrage, composée du commentaire des textes de Hume et de Budd, permet de situer les notions vues précédemment dans une perspective plus générale. Puisque la théorie humienne avait été présentée plus en détail auparavant, Zeimbekis propose un rappel de l'opposition entre conception relativiste (motivée par un constat empirique) et réaliste telle que présentée par Hume (p. 80-81). On insiste aussi sur les préoccupations «méta-esthétiques » de Hume, moins intéressé par la définition des concepts esthétiques que par une discussion autour de leur légitimité (p. 84) ainsi que sur les limites de la forme de réalisme proposée par Hume (p. 90). Le texte de Budd consiste pour sa part en un court extrait qui discute une interprétation contemporaine de Hume, coupé toutefois du contexte de discussion dans lequel il s'insérait. On cherche à y repousser le relativisme des jugements esthétiques en défendant l'existence de "principes esthétiques logiquement faibles» (p. 108). Le principal mérite de l'extrait choisi est de servir de prétexte à l'introduction de certaines figures contemporaines dont Alan Goldman et Eddy Zemach.

En conclusion, disons que cette publication relève le défi de rendre compte d'une problématique qui a une longue histoire tout en réussissant à résister à la tentation du parti pris et en sachant mettre en valeur la problématique. La commande implique en soi une grande neutralité de l'auteur, exigence qui a été respectée admirablement, d'autant plus que le format imposé par la collection oblige un traitement très schématique qui ajoute à la difficulté de la tâche. L'auteur a également su mettre en perspective les théories autour du jugement esthétique : bien que populaires chez les historiens qui s'intéressent à l'esthétique, ou dans leurs variantes contemporaines, elles ne sont pas les seules approches possibles, et la pertinence même de traiter de la notion de jugement esthétique a été remise en question par plusieurs. Notons que ce petit volume remplira sa fonction principalement auprès d'un lectorat déjà familier et sympathique aux théories issues de l'empirisme. Mais ce défaut a une contrepartie positive: on va à l'essentiel, ce qui permet de comprendre les enjeux de ce problème historique qui suscite toujours l'intérêt de plusieurs.

MÉLISSA THÉRIAULT

UQAM/Université de Provence

\section{Jean-François Filion, Dialectique et matière. La conceptualité incon- sciente des processus inorganiques dans la Philosophie de la nature (1830) de Hegel, Québec, Les Presses de l'Université Laval, 2007, 346 p.}

Cet ouvrage impose d'abord par son ambition. Reconnaissons d'emblée que la philosophie de la nature n'est ni la partie la plus connue du système hégélien ni encore moins la plus considérée. Or, dans cette étude, qui constitue une version remaniée de sa thèse de doctorat, Jean-François Filion se propose non pas simplement de réhabiliter la philosophie de la nature de Hegel — d'autres auteurs s’y sont déjà 
attelés de manière convaincante avant lui, notamment Vittorio Hösle dans sa monumentale étude Hegels System (1987) —, mais plutôt dans une démarche plus engagée, qui consiste à " prendre la spéculation au sérieux » (p. 3). Malgré les deux siècles qui nous séparent d'elle, la pensée hégélienne, pour peu qu'on prenne toute la mesure de sa « radicalité» (p. 2), peut encore nous aider à penser notre temps.

La thèse que défend J.-F. Filion est que la pensée spéculative hégélienne ouvre une piste de réflexion nouvelle et porteuse permettant de repenser la présente crise écologique. Comme on peut le lire, elle offre "des munitions à la révolution culturelle de l'écologisme » (p. 4). Selon l'auteur, toute l'originalité et la force de cette pensée tient $\mathrm{au}$ «renversement ontologique» (p. 317) qu'elle réussit à opérer quant au rapport entre l'être humain et la nature ; rapport dont d'aucuns, parmi les penseurs préoccupés par la question écologique, admettent qu'il s'agit là de la cause la plus profonde de la présente crise. En effet, au cœur de la menace qui guette l'intégrité écologique de l'humanité réside une conception de la nature, fruit d'une tradition métaphysique qui remonte en Occident au début des sciences modernes, qui voit dans celle-ci rien de plus qu'une simple matière brute, un matériau dont l'être humain peut disposer à sa guise pour la poursuite de ses propres fins. La nature n'est toujours considérée qu'en tant qu'altérité radicale, comme l'autre de l'humanité.

Or précisément, toute la philosophie spéculative de Hegel repose sur l'idée qu'il existe dans la nature une rationalité «inconsciente» (p. 23), une raison qui est et qui se donne à voir sous la forme manifeste d'une nature inorganique. Dans l'idéalisme absolu hégélien, la nature n'est point saisie en tant qu'altérité radicale, mais bien toujours en tant que simple moment d'un tout qui l'englobe, ce que Hegel nomme l' «Idée » : «La nature et l'esprit partagent le fait d'être les deux modes de la manifestation de l'Idée » (p. 112). Le réel naturel et le réel spirituel s'offrent dans la philosophie spéculative comme deux moments de la manifestation effective (wirkliches) de l'Idée. Autrement dit, "la clef interprétative de la conception hégélienne de la nature inorganique consiste à voir dans celle-ci la forme "aliénée" de la vie cosmique qui se désaliénera ici, sur Terre, avec l'apparition de la vie biologique et d'une espèce animale spéciale qui peut en parler» (p. 18). Aussi, par cette approche spéculative de la nature, la pensée hégélienne permet de surmonter l'aporie à laquelle est confrontée aujourd'hui toute pensée écologique.

À cette fin, Dialectique et matière s'articule sous la forme d'une lecture exégétique de la seconde partie de l'Encyclopédie des sciences philosophiques, celle qui précisément porte sur la philosophie de la nature, avec un accent mis sur les paragraphes 245 à 336. L'auteur a retenu pour cette lecture la troisième et dernière édition de l'ouvrage, celle de 1830, retravaillée par Hegel avant sa mort, et qui comprend de nombreuses "additions", soit les notes prises par les étudiants pendant ses leçons berlinoises. L'étude de J.-F. Filion s'organise autour de deux parties comportant au total sept chapitres. La première déploie une introduction à la philosophie de la nature chez Hegel avec une analyse de ce qui distingue cette pensée de celle de Friedrich Schelling, penseur à qui sera associée la figure de Hegel, jusqu'à la publication en 1806-1807 de son imposante Phénoménologie de l'esprit. La seconde partie s'emploie à expliciter la philosophie de la nature inorganique chez Hegel, soit les paragraphes 253 à 336.

La lecture que nous offre J.-F. Filion est conventionnelle — rien ici d'une lecture inédite - tout en étant néanmoins éclairante. Le travail de pensée ne se situe pas tant dans la lecture de Hegel comme telle que dans ce que cette lecture ouvre comme 
possibilité ; ce que précisément l'auteur parvient à très bien faire valoir en introduction et en conclusion de l'ouvrage.

Cependant, malgré les grands mérites de cette démarche et la rigueur avec laquelle elle est menée, on ne peut s'empêcher de se demander à quel lectorat un tel ouvrage est destiné. En effet, si cette étude s'adresse avant tout à ceux désireux de trouver dans la pensée spéculative hégélienne une piste d'espoir pour sortir l'humanité de la crise écologique - objectif que l'auteur semble privilégier dans l'introduction — alors, force est d'admettre qu'il manque à cet ouvrage une introduction générale à la pensée de Hegel dans son ensemble. Assurément, un lecteur néophyte risque en de nombreux passages de n'y rien comprendre tant la pensée hégélienne, et peut-être encore plus sa philosophie de la nature, manifeste une impénétrabilité certaine.

Par delà cet élément de critique, ou peut-être même pour y parer, il ne reste maintenant qu'à souhaiter que Jean-François Filion poursuive sur la voie ouverte par cette étude, en mettant à profit l'approche spéculative hégélienne afin de proposer des pistes de solutions pour sortir d'humanité de la présente menace écologique qui pèse sur son habitat. Rendre justice à la profondeur du hégélianisme peut parfois exiger de passer sous silence la pensée du maître.

DANIC PARENTEAU

École d'études politiques, Université d'Ottawa

\section{Alain Beaulieu (dir.), Gilles Deleuze, héritage philosophique, Paris, PUF, 2005, 174 pages.}

L'année 2005 a marqué le dixième anniversaire de la mort de Gilles Deleuze. À l'occasion d'une publication dans la collection «Débats philosophiques " des Presses Universitaires de France, Alain Beaulieu entend défendre l'idée que Gilles Deleuze fut "simultanément historien, créateur et expérimentateur de concepts" (p. 12). En refusant de dissocier catégoriquement et d'étudier séparément - comme on l'avait généralement fait jusque-là — ces différentes facettes de la vie intellectuelle du philosophe, Alain Beaulieu espère donner un second souffle à la réception posthume de ses écrits. À cette fin, il a réuni dans ce volume les contributions de six des principaux commentateurs de l'œuvre deleuzienne, à la fois d'Europe (Manola Antonioli, Arnaud Villani, Olivier Fahle et Stéfan Leclercq) et de ce côté-ci de l'Atlantique (Constantin Boundas et Alain Beaulieu).

\section{1.}

Avec son analyse des "Stratégies différentielles dans la pensée deleuzienne ", Constantin Boundas cherche à dégager les réquisits et les conséquences d'une authentique philosophie de la différence. L'auteur nous rappelle tout d'abord que, chez Deleuze, «la différence ne se donne pas comme un concept» (p. 16) puisqu'elle est pensée comme un processus, ce qui implique en retour une ontologie des forces et des relations qui s'oppose à l'étude des différents étants dans leur particularité. Certes, les forces se manifestent nécessairement dans des états de choses distincts les uns des autres, mais puisque ceuxci ne peuvent rendre compte de leur devenir et du processus dont résulte leur différenciation, Deleuze en est conduit à penser, à la suite de Bergson, une différentiation virtuelle 